International Journal of Pure and Applied Mathematics

Volume 89 No. 3 2013, 353-356

ISSN: 1311-8080 (printed version); ISSN: 1314-3395 (on-line version)

url: http://www.ijpam.eu

doi: http://dx.doi.org/10.12732/ijpam.v89i3.6

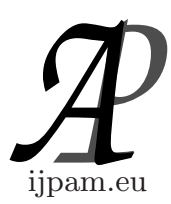

\title{
A POINTWISE POISSON APPROXIMATION FOR RANDOM SUMS OF GEOMETRIC RANDOM VARIABLES
}

\author{
K. Teerapabolarn \\ Department of Mathematics \\ Faculty of Science \\ Burapha University \\ Chonburi, 20131, THAILAND
}

\begin{abstract}
We determine a bound for the point metric between the distribution of random sums of independent geometric random variables and an appropriate Poisson distribution. Three examples have been given to illustrate the results obtained.
\end{abstract}

AMS Subject Classification: 62E17, 60F05, 60G05

Key Words: geometric random variable, Poisson approximation, random sums, point metric

\section{Introduction}

Let $X_{1}, \ldots, X_{n}$ be $n$ independently distributed geometric random variables, each with $P\left(X_{i}=k\right)=\left(1-p_{i}\right)^{k} p_{i}, k=0,1, \ldots$, and let $S_{n}=\sum_{i=1}^{n} X_{i}$ and $U_{\lambda}$ a Poisson random variable with mean $\lambda$. It is well-known that if all $q_{i}=1-p_{i}$ are small, then the distribution of $S_{n}$ can be approximated by a Poisson distribution with mean $\lambda$. Correspondingly, the probability function of $S_{n}$, denoted by $\mathrm{P}_{S_{n}}\left(x_{0}\right)$, can also be approximated by an appropriate Poisson probability function with mean $\lambda$, denoted by $\wp_{\lambda_{n}}\left(x_{0}\right)$, where $x_{0} \in \mathbb{N} \cup\{0\}$. In this case, Teerapabolarn [1] gave a bound for the point metric between the distributions

Received: June 18, 2013

(c) 2013 Academic Publications, Ltd. url: www.acadpubl.eu 
of $S_{n}$ and $U_{\lambda_{n}}$ as follows:

$$
\left|\mathrm{P}_{S_{n}}\left(x_{0}\right)-\wp_{\lambda_{n}}\left(x_{0}\right)\right| \leq \sum_{i=1}^{n} \min \left\{\frac{1-e^{-\lambda_{n}}}{\lambda_{n}}, \frac{1}{x_{0}}, p_{i}\right\} \frac{q_{i}^{2}}{p_{i}}
$$

for $x_{0} \in \mathbb{N}$ and $\lambda_{n}=\sum_{i=1}^{n} q_{i}$.

Let us consider the case where $n$ is a random variable (not a fixed number), that is, if $N$ is a non-negative integer valued random variable and independent of the $X_{i}$ 's, then the sums $S_{N}=\sum_{i=1}^{N} X_{i}$ is called random sums of independent geometric random variables, which makes it more interesting. In this study, we are interest to approximate $\mathrm{P}_{S_{N}}\left(x_{0}\right)$ by $\wp_{\lambda}\left(x_{0}\right)$ when $\lambda=E\left(\lambda_{N}\right)=\sum_{i=1}^{N} q_{i}$.

\section{Result}

A bound for the point metric of $\mathrm{P}_{S_{N}}\left(x_{0}\right)$ and $\wp_{\lambda}\left(x_{0}\right)$ is presented as follows.

Theorem 2.1. For $x_{0} \in \mathbb{N}$ and $\lambda=E\left(\lambda_{N}\right)=\sum_{i=1}^{N} q_{i}$, then we have

$$
\begin{aligned}
& \left|\mathrm{P}_{S_{N}}\left(x_{0}\right)-\wp_{\lambda}\left(x_{0}\right)\right| \leq \min \left\{\frac{1-e^{-\lambda}}{\lambda}, \frac{1}{x_{0}}\right\} E\left|\lambda_{N}-\lambda\right| \\
& \quad+\min \left\{E\left(\frac{1-e^{-\lambda_{N}}}{\lambda_{N}} \sum_{i=1}^{N} \frac{q_{i}^{2}}{p_{i}}\right), \frac{1}{x_{0}} E\left(\sum_{i=1}^{N} \frac{q_{i}^{2}}{p_{i}}\right), E\left(\sum_{i=1}^{N} q_{i}^{2}\right)\right\},
\end{aligned}
$$

where $\mathrm{P}_{S_{N}}(0)=E\left(\prod_{i=1}^{N} p_{i}\right)$.

Proof. From the fact that

$$
\begin{aligned}
\left|\mathrm{P}_{S_{N}}\left(x_{0}\right)-\wp_{\lambda}\left(x_{0}\right)\right| \leq & \sum_{n=0}^{\infty} P(N=n)\left|\mathrm{P}_{S_{n}}\left(x_{0}\right)-\wp_{\lambda_{n}}\left(x_{0}\right)\right| \\
& +\left|\wp_{\lambda_{N}}\left(x_{0}\right)-\wp_{\lambda}\left(x_{0}\right)\right| .
\end{aligned}
$$

Following the results in (1.1) and [2], we have

$$
\begin{aligned}
\left|\wp_{\lambda_{N}}\left(x_{0}\right)-\wp_{\lambda}\left(x_{0}\right)\right| \leq & \sum_{n=0}^{\infty} P(N=n) \sum_{i=1}^{n} \min \left\{\frac{1-e^{-\lambda_{n}}}{\lambda_{n}}, \frac{1}{x_{0}}, p_{i}\right\} \frac{q_{i}^{2}}{p_{i}} \\
& +\min \left\{\frac{1-e^{-\lambda}}{\lambda}, \frac{1}{x_{0}}\right\} E\left|\lambda_{N}-\lambda\right|,
\end{aligned}
$$

which yields (2.1). 
If $X_{i}$ 's are identically distributed, then the following corollary is an immediately consequence of the Theorem 2.1

Corollary 2.1. For $x_{0} \in \mathbb{N}$, if $p_{1}=p_{2}=\cdots=p$, then we have the following:

$$
\begin{aligned}
\left|\mathrm{P}_{S_{N}}\left(x_{0}\right)-\wp_{\lambda}\left(x_{0}\right)\right| \leq & \min \left\{\frac{1-e^{-q E(N)}}{q E(N)}, \frac{1}{x_{0}}\right\} q E|N-E(N)| \\
& +\min \left\{\frac{q}{p} E\left(1-e^{-q N}\right), \frac{q^{2}}{p x_{0}} E(N), q^{2} E(N)\right\},
\end{aligned}
$$

where $\mathrm{P}_{S_{N}}(0)=p E(N)$.

\section{Examples}

This section, we give three examples to illustrate the result in the case where $X_{i}$ 's are identically distributed.

Example 3.1. For $n(n \in \mathbb{N})$ is fixed, let $N$ be a positive integer-valued random variable with probability function

$$
P(N=k)= \begin{cases}\frac{1}{2} & , k=n \\ \frac{1}{2} & , k=2 n \\ 0 & , \text { otherwise. }\end{cases}
$$

Therefore $E(N)=\frac{3 n}{2}$ and $E|N-E(N)|=\frac{n}{2}$. Let $p_{1}=p_{2}=\cdots=p$, then we have

$$
\left|\mathrm{P}_{S_{N}}\left(x_{0}\right)-\wp_{\lambda}\left(x_{0}\right)\right| \leq \min \left\{\frac{1-e^{-\frac{3 n q}{2}}}{3}, \frac{n q}{2 x_{0}}\right\}+\min \left\{\frac{q}{p}, \frac{3 n q^{2}}{2 p x_{0}}, \frac{3 n q^{2}}{2}\right\},
$$

where $x_{0} \in \mathbb{N}$ and $\mathrm{P}_{S_{N}}(0)=\frac{3 n p}{2}$.

Example 3.2. Let $N$ be a positive integer-valued random variable with probability function

$$
P(N=n)=\frac{1}{2^{n}}, n=1,2, \ldots,
$$

then we have $E(N)=2$ and $E|N-E(N)|=1$. If $p_{1}=p_{2}=\cdots=p$, then we obtain

$$
\left|\mathrm{P}_{S_{N}}\left(x_{0}\right)-\wp_{\lambda}\left(x_{0}\right)\right| \leq \min \left\{\frac{1-e^{-2 q}}{2}, \frac{q}{x_{0}}\right\}+\min \left\{\frac{q}{p}, \frac{2 q^{2}}{p x_{0}}, 2 q^{2}\right\},
$$


where $x_{0} \in \mathbb{N}$ and $\mathrm{P}_{S_{N}}(0)=2 p$.

Example 3.3. Let $N$ has a Poisson random variable with probability function

$$
P(N=n)=\frac{e^{-\mu} \mu^{n}}{n !}, n=0,1, \ldots,
$$

then we have $E(N)=\mu$ and $E|N-E(N)|=2 \mu e^{-\mu}$. If $p_{1}=p_{2}=\cdots=p$, then we obtain

$$
\left|\mathrm{P}_{S_{N}}\left(x_{0}\right)-\wp_{\lambda}\left(x_{0}\right)\right| \leq \min \left\{1-e^{-\mu q}, \frac{\mu q}{x_{0}}\right\} 2 e^{-\mu}+\min \left\{\frac{q}{p}, \frac{\mu q^{2}}{p x_{0}}, \mu q^{2}\right\},
$$

where $x_{0} \in \mathbb{N}$ and $\mathrm{P}_{S_{N}}(0)=\mu p$.

\section{References}

[1] K. Teerapabolarn, A pointwise approximation for independent geometric random variables, Int. J. Pure Appl. Math., 76 (2012), 727-732.

[2] K. Teerapabolarn, A new bound on pointwise Poisson approximation for random sums of Bernoulli random variables, Int. J. Pure Appl. Math., 89, No. 2 (2013), doi: 10.12732/ijpam.v89i2.2. 